\title{
Impairment in Quantitative Microvascular Function in Non-Ischemic Cardiomyopathy as Demonstrated Using Cardiovascular Magnetic Resonance
}

Jeremy A. Slivnick ( $\square$ jeremy.slivnick@osumc.edu )

The Ohio State University Wexner Medical Center https://orcid.org/0000-0002-2327-4544

Karolina M. Zareba

The Ohio State University Wexner Medical Center

Vien T. Truong

The Christ Hospital Physicians Ohio Heart \& Vascular

Ellen Liu

The Ohio State University Wexner Medical Center

\section{Alexis Barnes}

The Ohio State University Wexner Medical Center

\section{Wojciech Mazur}

Christ Hospital Physicians Ohio Heart and Vascular: The Christ Hospital Physicians Ohio Heart \& Vascular

\section{Philip Binkley}

The Ohio State University Wexner Medical Center

\section{Research Article}

Keywords: Cardiac Magnetic Resonance Imaging (MRI), Imaging, Cardiomyopathy, Heart Failure

Posted Date: May 10th, 2021

DOI: https://doi.org/10.21203/rs.3.rs-496006/v1

License: (a) (1) This work is licensed under a Creative Commons Attribution 4.0 International License. Read Full License 


\section{Abstract \\ Purpose}

Microvascular dysfunction (MVD) - defined as impaired augmentation of the microcirculation in response to stress-is present in various cardiovascular diseases and portends worse outcomes. We aimed to evaluate the relationship between MVD and non-ischemic cardiomyopathy (NICM) utilizing stress cardiovascular magnetic resonance (CMR) as compared to a cohort of control patients.

\section{Methods}

We retrospectively studied 41 consecutive patients with NICM (mean age $51 \pm 14,59 \%$ male) and 58 controls with preserved systolic function (mean age $51 \pm 13,31 \%$ male) who underwent adenosine stress CMR exams between 2011-2016. Microvascular function was assessed visually and with myocardial perfusion reserve index (MPRI), quantified using first pass perfusion imaging by comparing perfusion slopes of myocardium and blood pool at rest/stress. MVD was defined visually as presence of subendocardial stress perfusion defect and quantitatively by MPRI $<1.51$. MPRI was compared between NICM and controls using univariate analysis and multivariable linear regression.

\section{Results}

Impaired MPRI was noted in 37 patients (23 in NICM and 14 in control cohorts). In patients with NICM, 23 $(56 \%)$ had MVD by quantitative assessment, while 11 (27\%) by visual evaluation. No differences in comorbidities were noted between cohorts. Compared with controls, NICM patients had lower rest perfusion slope (3.9 vs 4.9, $p=0.05$ ), stress perfusion slope (8.8 vs $11.7, p<0.001)$, and MPRI (1.41 vs $1.74, p=0.02)$. MPRI remained associated with NICM after controlling for age, gender, hypertension, diabetes, and late gadolinium enhancement $(\log$ MPR, $\beta$ coefficient $=-0.17, p=0.009)$.

\section{Conclusions}

MVD assessed with stress CMR is highly prevalent in NICM as compared to control patients with preserved systolic function. Quantitative MPRI assessment identities more NICM patients with MVD as compared to visual evaluation. NICM remains independently associated with an impaired MPRI after controlling for covariates. Further studies are needed to determine whether targeted therapies to treat MVD are beneficial in NICM.

\section{Introduction}

Coronary microvascular disease (MVD) - defined as impaired augmentation of coronary circulation with ischemia in response to stress in the absence of epicardial coronary artery disease (CAD)-afflicts a 
significant proportion of patients across a range of cardiovascular disorders and is associated with adverse outcomes ${ }^{1,2}$. In a healthy individual, vasodilation of small vessel arterioles in response to stress allows for augmentation of the coronary microcirculation by as much as $4-5$ times its original value ${ }^{3}$. This process is adaptive and allows for the delivery of increased coronary blood flow to meet increased demand in response to physiologic stress. However, stress-induced microcirculatory response may be reduced by more than half in the presence of MVD. The pathophysiology of MVD is thought to be multifactorial and related to small vessel atherosclerosis, vascular rarefaction, and endothelial dysfunction ${ }^{4}$.

MVD can be assessed using invasive and non-invasive techniques. Microvascular reactivity can be evaluated with invasive coronary angiography (ICA) using coronary flow reserve (CFR) or the index of myocardial resistance (IMR) in response to a stress agent ${ }^{5}$. However, these invasive methods harbor procedural risks. Non-invasively, microvascular perfusion reserve can be assessed with positron emission tomography (PET) or cardiovascular magnetic resonance (CMR). CMR takes advantage of first pass gadolinium perfusion imaging in order to quantify semi-quantitative or fully quantitative changes in perfusion in response to a stress agent-typically adenosine. CMR-derived myocardial perfusion reserve index (MPRI) has been shown to correlate with CFR on ICA ${ }^{6}$.

MVD is increasingly recognized as a cause of angina and chest pain syndromes in the absence of obstructive CAD. In a 189 female subset of the Women's Ischemia Syndrome Evaluation (WISE) study, MVD was present in $39 \%$ of patients on invasive adenosine provocation and portended increased rates of major adverse cardiovascular events (MACE) ${ }^{7}$. Based on this and other studies, MVD is theorized as a mechanism to explain increased rates of MACE in patients with angina in the absence of epicardial CAD in comparison to the general population 7,8 . MVD has also been demonstrated in association with other conditions including hypertension, dyslipidemia, and diabetes ${ }^{9-12}$.

Non-ischemic cardiomyopathy (NICM) is responsible for up to $40 \%$ of cases of systolic heart failure and carries a poor prognosis not dissimilar to its ischemic counterpart ${ }^{13-16}$. MVD represents one potential driver of MACE in this population. In a retrospective analysis by Majmudar et al, MVD as assessed with positron emission tomography (PET) was highly prevalent in NICM and was associated with increased $\mathrm{MACE}^{2}$. Little is known whether CMR is useful for assessing MVD in NICM. A study of 28 patient by Bell et a/demonstrated that MPR was more prevalent in NICM as compared with healthy controls ${ }^{17}$. Stress CMR is commonly obtained in the evaluation of newly diagnosed NICM due to its ability to exclude alternative etiologies of cardiomyopathy such as ischemia, infiltrative heart disease, storage disorders, and myocarditis. Expansion of CMR to include the non-invasive assessment of MVD in NICM would increase its diagnostic and prognostic capabilities.

We aimed to evaluate the relationship between MVD and NICM utilizing adenosine stress CMR as compared to a cohort of control patients with preserved systolic function. 


\section{Materials And Methods}

\section{Study Participants}

We retrospectively identified 41 patients with NICM and 58 control patients with preserved systolic function and without clinical heart failure who had undergone comprehensive adenosine stress CMR perfusion between 2011 and 2016 at a single academic institution. NICM was defined by left ventricular ejection fraction (LVEF) $₫ 50 \%$ without significant CAD. Coronary artery disease was defined as the presence of $₫ 50 \%$ coronary artery stenosis on invasive or CT coronary angiography, prior coronary artery revascularization, the presence of a segmental perfusion defect on CMR, or the presence of infarct scar on late gadolinium enhancement (LGE) imaging. A control cohort was identified by selecting patients with LVEF $\triangle 50 \%$ who were referred for clinical stress CMR exam. Control patients were excluded if they had any prior history of obstructive CAD on coronary angiography, prior coronary artery revascularization, prior myocardial infarction, or a history of heart failure. Control patients were selected to match risk factors for microvascular dysfunction. Additionally, patients with infarct scar by LGE or focal perfusion defects on first pass stress perfusion were excluded. In the control cohort patients, the indication for stress CMR was chest pain in $38(66 \%)$, ventricular ectopy or ventricular tachycardia in 7 (12\%), dyspnea in $3(5 \%)$, syncope in $2(3 \%)$, and other in $8(14 \%)$ patients.

Clinical patient characteristics and comorbidities were established through a review of the electronic medical record. The following baseline clinical characteristics were collected: age, gender, ethnicity, body mass index, New York Heart Association Class, NICM etiology, serum creatinine, hematocrit, BNP, and troponin. The Ohio State University Institutional Review Board approved this retrospective study and agreed to waive informed consent. All investigators have full access to the data and take responsibility for its integrity and the data analysis.

\section{CMR Imaging and Analysis:}

Patients underwent clinical CMR exams using a 1.5 Tesla scanner (Magnetom Avanto or Espree, Siemens Medical Solutions, Erlangen, Germany). LV volumes, mass, and EF were assessed using steady state free precession (SSFP) sequences. Ventricular volumes and function were quantified from endocardial and epicardial tracing of serial short axis slices at end diastole and end systole. LV mass was calculated by multiplying the total myocardial volume at end diastole by the specific gravity of the myocardium (1.05 $\mathrm{g} / \mathrm{ml})^{18}$.

Vasodilator stress CMR was performed using a $140 \mathrm{mcg} / \mathrm{kg} / \mathrm{min}$ adenosine infusion for 2 minutes prior to first-pass perfusion imaging, and continued until completion of the perfusion imaging data acquisition. First-pass perfusion imaging was performed using a $0.05 \mathrm{mmol} / \mathrm{kg}$ bolus of gadolinium based contrast agent (GBCA). A rest perfusion study was performed using the same protocol. Myocardial perfusion defects were assessed by both visual and quantitative analysis. Quantification was performed by 
manually delineating endocardial and epicardial left ventricular borders in the mid-short axis slice during both stress and rest first-pass perfusion with care to exclude blood pool activity (Cvi42, Circle Cardiovascular Imaging, Calgary, Canada). Only mid-short axis slices were used out of concern for partial volume effects related to thin distal segments in the NICM group. Signal intensity curves of segmental myocardium were automatically generated. MPRI was defined as: MPRI = RUstress/RUrest. RU is defined as the ratio between the maximum upslope of the first-pass myocardial perfusion time-intensity curve divided by the maximum upslope of the first-pass LV cavity time-intensity curve (Figure 1). MVD was defined quantitatively as MPRI $<1.51$ which was the lower interquartile range for the entire cohort and is similar to that used in the WISE subanalysis ${ }^{19}$. Qualitative MVD was defined as the presence of a circumferential subendocardial perfusion defect on first pass stress imaging ${ }^{20}$. Per guidelines, defects which occurred prior to contrast arrival in the LV myocardium, persisted $<10$ heart beats, or were $<2$ pixels wide were considered to be due to dark rim artifact and were not identified as true perfusion defects ${ }^{21}$.

Late gadolinium enhancement imaging was performed using gradient-echo inversion recovery sequences and phase sensitive inversion recovery (PSIR) reconstructions 10 minutes after administration of an additional $0.1 \mathrm{mmol} / \mathrm{kg}$ of GBCA ${ }^{22}$. The presence of LGE was assessed by 2 expert level 3 trained operators blinded to clinical data and had to be present in either two consecutive short axis slices or in two orthogonal imaging planes. LGE was scored according to its presence and extent based on the number of American Heart Association segments ${ }^{23}$.

\section{Statistical analysis}

Categorical data are presented as frequency with percentage, and comparisons between groups were performed using the chi-square test or Fisher exact test. Skewness, kurtosis, and visual inspection of the histogram and QQ plot were checked to assess the distribution of continuous variables. Continuous variables are presented as mean \pm standard deviation (SD) for normal distribution or expressed as median (interquartile range) for non-normal distribution. Continuous variables were compared using Student's t-test or the Wilcoxon rank-sum test, as appropriate. Univariate and multivariable linear regression was performed to assess the relationship between the presence of NICM and MPRI after controlling for significant covariates. Because of non-normal distributed residual in multivariable analysis, logarithmic transformation was performed. To test the robustness of association of nonischemic cardiomyopathy and RPP, the bootstrap method with 2,000 resampling technique was performed to estimate $95 \%$ bias-corrected and accelerated confidence intervals. Further, gamma regression model with an identity link function was applied to assess the robustness of result. Regression diagnostics were performed to test model assumptions. Statistical analyses were performed using $\mathrm{R}$ software, version 4.03 (The R Foundation, Vienna, Austria).

\section{Results}

\section{Demographics:}


In total, 41 patients with NICM (mean age $51 \pm 14,59 \%$ male) and 58 controls (mean age $51 \pm 13,31 \%$ male) were identified. Etiologies of NICM are displayed in Figure 2. Of the patients with NICM, 22 (54\%) were NYHA Class I, 12 (29\%) were Class II, and 7 (17\%) belonged to Class III. No patients endorsed NYHA Class IV symptoms. Clinical characteristics of the NICM and control cohorts are presented in Table 1. Compared with controls, patients with NICM were more likely to be male gender and of African American ethnicity (Table 1). There were no significant differences in rates of classic cardiovascular risk factors (diabetes, hypertension, hyperlipidemia) or atrial arrhythmias between groups.

\section{CMR Characteristics}

A comparison of CMR parameters is presented in Table 1. Compared with controls, patients with NICM had significantly higher left atrial volumes, LV volumes and mass, and lower LVEF. Late gadolinium enhancement was significantly more prevalent in the NICM vs control cohorts $(51 \%$ vs $16 \%, p<0.001)$. LGE patterns included midwall and/or epicardial enhancement as patients with subendocardial or transmural infarct scar were excluded.

First pass perfusion analysis revealed that patients with NICM has significantly lower MPR slopes both at rest (3.9 vs $4.9, p=0.045)$ and with stress ( 8.8 vs $11.7, p<0.001)$ when compared to the control group. MPRI was significantly lower in the NICM group as compared with controls $(1.41 \mathrm{vs} \mathrm{1.74,p=0.02)}$ (Figure 3). A density plot showing the distribution of MPRI for NICM and control cohorts is displayed in Figure 4. In the NICM cohort, 23 (56\%) patients had MVD by quantitative analysis (MPRI<1.51) while only 11 (27\%) by visual analysis. In the control cohort, 14 (24\%) patients had MVD by quantitative analysis while none were noted to have MVD by visual criteria.

\section{Comparison of Normal versus Impaired MPR}

In total there were 37 patients in the entire cohort with MVD by quantitative analysis. A comparison of patients with impaired vs. normal MPRI is presented in Table 2. Patients with impaired MPRI were more likely to be hypertensive as compared to those with normal MPI. There was a trend towards older age and more diabetes in impaired MPRI cohort. With respect to CMR parameters, patients with impaired MPRI had significantly lower LVEF and higher indexed LV mass.

\section{Multivariable Analysis}

In unadjusted analysis, compared to control group, patients with non-ischemic cardiomyopathy had significantly lower MPRI (log MPR, $\beta$ coefficient = -0.14, p=0.03). After adjusting for age, gender, diabetes, hypertension, and presence of LGE, non-ischemic cardiomyopathy remained independently associated with lower MPRI (log MPR, $\beta$ coefficient = -0.17, $p=0.009$ ) (Table 3, Figure 4). Bootstrap method and gamma regression confirmed that there was a significant association between non-ischemic cardiomyopathy and lower MPRI (Table 3, Supplemental Table). Additionally, the presence of hypertension was also independently associated with MVD. 


\section{Discussion}

We evaluated the relationship between MVD in patients with NICM as compared with control patients with preserved LV function. Three key points can be inferred from our analysis: 1) MVD is more prevalent in patients with $\mathrm{NICM}$ as compared to controls, 2) quantitative CMR evaluation of MVD remains more robust than visual inspection, 3 ) NICM remains independently associated with an impaired MPRI after controlling for covariates.

In our cohort of patients with NICM over half exhibited impaired myocardial perfusion reserve index. The prevalence of MVD in NICM was doubled as compared to our control cohort. This parallels findings from other studies which previously identified a high prevalence of MVD in other disease entities including hypertension, hyperlipidemia, diabetes, and in women with chest pain syndromes ${ }^{7,9-12}$. In our cohort, quantitative analysis was more sensitive for detecting MVD as compared with qualitative analysis. This is consistent with prior CMR based studies which have identified MVD even in the absence of visual perfusion defects in patients with cardiac syndrome $\mathrm{X}^{24}$.

We demonstrate a robust relationship between the presence of NICM and CMR-derived MPRI even after controlling for clinical and CMR covariates. Our study parallels previous findings by Majmudar and Bell et $a^{2,17}$. We validate CMR findings from Bell et al who previously demonstrated impaired MPRI in a prospective cohort of 13 patients with NICM as compared with 15 control subjects ${ }^{17}$. An important aspect of our study is the ability to adjust for clinical and CMR covariates given the known link between MVD and hypertension, diabetes, and gender ${ }^{9,11}$. We also note this significant relationship between hypertension and MVD.

The presence of MVD may have important implications in patients with NICM. MVD may significantly contribute to exercise-related symptoms in patients with NICM. In comparison to controls, patients with microvascular angina exhibit greater impairments in diastolic function in response to adenosine $\mathrm{e}^{25}$. Additionally, the presence of MVD is associated with impaired exercise tolerance in heart failure with preserved EF (HFpEF) patients ${ }^{26}$. This may explain in part why some heart failure patients experience symptoms with exercise in the absence of elevated filling pressures ${ }^{27}$. The pathogenesis behind this relationship is currently unclear, and whether MVD leads to NICM or vice versa is debated. One theory proposed in the HFpEF population is that chronic inflammation driven by comorbidities results in endothelial dysfunction and depletion in nitric oxide (NO), ultimately resulting in MVD ${ }^{28}$. Further research is needed to determine whether improved treatment of comorbidities or pharmacotherapies targeting the microvasculature can be beneficial in NICM patients.

\section{Limitations}


Our study is limited by its retrospective nature and therefore may be influenced by unknown confounders. The control group was identified retrospectively amongst patients referred for clinical CMR exam. The relatively high prevalence of diabetes, hypertension, hyperlipidemia, and atrial arrhythmias are reflective of a control group at high risk for MVD and matched comorbidities of the DCM group. The presence of significant MPRI differences despite matched MVD risk factors further underscores perfusion abnormalities in DCM, beyond what could be expected by traditional MVD risk factors. As this is a retrospective CMR-based study evaluating MPRI, invasive correlation was not obtained.

\section{Conclusion}

In conclusion, MVD as assessed quantitatively with CMR is highly prevalent amongst patients with NICM as compared to control patients with preserved systolic function. Quantitative MPRI assessment identities more patients with MVD as compared to visual evaluation. NICM remains independently associated with an impaired MPRI after controlling for both clinical and CMR covariates. The expansion of stress CMR to include the evaluation of MVD will allow for improved phenotyping of NICM patients which may lead to better tailored therapies.

\section{Declarations}

Funding: No grant or industry funding was utilized for the purposes of this study.

Conflicts of interest/Competing interests: All authors have reported that they have no relationships relevant to the contents of this paper to disclose.

Availability of data and material: If requested, the authors are agreeable to provide a de-identified copy of the data to the editorial board.

Code Availability: If requested, the authors agree to provide all software applications utilized for the purposes of this study.

Ethics Approval: This study was approved by the Ohio State University Institutional Review Board who has agreed to waive informed consent for the purposes of this study.

\section{References}

1. Murthy VL, Naya M, Taqueti VR et al (2014) Effects of sex on coronary microvascular dysfunction and cardiac outcomes. Circulation Jun 129(24):2518-2527.

doi:10.1161/CIRCULATIONAHA.113.008507

2. Majmudar MD, Murthy VL, Shah RV et al (2015) Quantification of coronary flow reserve in patients with ischaemic and non-ischaemic cardiomyopathy and its association with clinical outcomes. Eur Heart J Cardiovasc Imaging Aug 16(8):900-909. doi:10.1093/ehjci/jev012 
3. Sdringola S, Johnson NP, Kirkeeide RL, Cid E, Gould KL (2011) Impact of unexpected factors on quantitative myocardial perfusion and coronary flow reserve in young, asymptomatic volunteers. JACC Cardiovasc Imaging Apr 4(4):402-412. doi:10.1016/j.jcmg.2011.02.008

4. Taqueti VR, Di Carli MF (2018) Coronary Microvascular Disease Pathogenic Mechanisms and Therapeutic Options: JACC State-of-the-Art Review. J Am Coll Cardiol 11(21):2625-2641. doi:10.1016/j.jacc.2018.09.042 72 ) .

5. Lee BK, Lim HS, Fearon WF et al (2015) Invasive evaluation of patients with angina in the absence of obstructive coronary artery disease. Circulation Mar 131(12):1054-1060. doi:10.1161/CIRCULATIONAHA.114.012636

6. Kurita T, Sakuma H, Onishi K et al (2009) Regional myocardial perfusion reserve determined using myocardial perfusion magnetic resonance imaging showed a direct correlation with coronary flow velocity reserve by Doppler flow wire. Eur Heart J Feb 30(4):444-452. doi:10.1093/eurheartj/ehn521

7. Pepine CJ, Anderson RD, Sharaf BL et al (Jun 2010) Coronary microvascular reactivity to adenosine predicts adverse outcome in women evaluated for suspected ischemia results from the National Heart, Lung and Blood Institute WISE (Women's Ischemia Syndrome Evaluation) study. J Am Coll Cardiol 55(25):2825-2832. doi:10.1016/j.jacc.2010.01.054

8. Jespersen L, Hvelplund A, Abildstrøm SZ et al (2012) Stable angina pectoris with no obstructive coronary artery disease is associated with increased risks of major adverse cardiovascular events. Eur Heart J Mar 33(6):734-744. doi:10.1093/eurheartj/ehr331

9. Di Carli MF, Janisse J, Grunberger G, Ager J (Apr 2003) Role of chronic hyperglycemia in the pathogenesis of coronary microvascular dysfunction in diabetes. J Am Coll Cardiol 41(8):13871393. doi:10.1016/s0735-1097(03)00166-9

10. Pitkänen OP, Raitakari OT, Niinikoski H et al (Dec 1996) Coronary flow reserve is impaired in young men with familial hypercholesterolemia. J Am Coll Cardiol 28(7):1705-1711. doi:10.1016/S07351097(96)00376-2

11. Laine H, Raitakari OT, Niinikoski H et al (Jul 1998) Early impairment of coronary flow reserve in young men with borderline hypertension. J Am Coll Cardiol 32(1):147-153. doi:10.1016/s07351097(98)00222-8

12. Quercioli A, Pataky Z, Montecucco F et al (2012) Coronary vasomotor control in obesity and morbid obesity: contrasting flow responses with endocannabinoids, leptin, and inflammation. JACC Cardiovasc Imaging Aug 5(8):805-815. doi:10.1016/j.jcmg.2012.01.020

13. Cohn JN, Tognoni G, Investigators VHFT (Dec 2001) A randomized trial of the angiotensin-receptor blocker valsartan in chronic heart failure. N Engl J Med 345(23):1667-1675. doi:10.1056/NEJMoa010713

14. McMurray JJ, Packer M, Desai AS et al (Sep 2014) Angiotensin-neprilysin inhibition versus enalapril in heart failure. N Engl J Med 371(11):993-1004. doi:10.1056/NEJMoa1409077

15. Zannad F, McMurray JJ, Krum H et al (Jan 2011) Eplerenone in patients with systolic heart failure and mild symptoms. N Engl J Med 364(1):11-21. doi:10.1056/NEJMoa1009492 
16. Lee DS, Gona P, Vasan RS et al (2009) Relation of disease pathogenesis and risk factors to heart failure with preserved or reduced ejection fraction: insights from the framingham heart study of the national heart, lung, and blood institute. Circulation Jun 119(24):3070-3077. doi:10.1161/CIRCULATIONAHA.108.815944

17. Bell SP, Adkisson DW, Ooi H, Sawyer DB, Lawson MA, Kronenberg MW (2013) Impairment of subendocardial perfusion reserve and oxidative metabolism in nonischemic dilated cardiomyopathy. J Card Fail Dec 19(12):802-810. doi:10.1016/j.cardfail.2013.10.010

18. Reiter G, Reiter U, Rienmüller R, Gagarina N, Ryabikin A (2004) On the value of geometry-based models for left ventricular volumetry in magnetic resonance imaging and electron beam tomography: a Bland-Altman analysis. Eur J Radio/ Nov 52(2):110-118. doi:10.1016/j.ejrad.2003.10.003

19. Thomson LE, Wei J, Agarwal $\mathrm{M}$ et al. Cardiac magnetic resonance myocardial perfusion reserve index is reduced in women with coronary microvascular dysfunction. A National Heart, Lung, and Blood Institute-sponsored study from the Women's Ischemia Syndrome Evaluation. Circ Cardiovasc Imaging Apr 2015;8(4)doi:10.1161/CIRCIMAGING.114.002481

20. Panting JR, Gatehouse PD, Yang GZ et al (2002) Abnormal subendocardial perfusion in cardiac syndrome $X$ detected by cardiovascular magnetic resonance imaging. N Engl J Med Jun 346(25):1948-1953. doi:10.1056/NEJMoa012369

21. Schulz-Menger J, Bluemke DA, Bremerich J et al (2020) Standardized image interpretation and postprocessing in cardiovascular magnetic resonance - 2020 update: Society for Cardiovascular Magnetic Resonance (SCMR): Board of Trustees Task Force on Standardized Post-Processing. J Cardiovasc Magn Reson 03(1):19. doi:10.1186/s12968-020-00610-6 22 ) .

22. Kellman P, Arai AE, McVeigh ER, Aletras AH (2002) Phase-sensitive inversion recovery for detecting myocardial infarction using gadolinium-delayed hyperenhancement. Magn Reson Med Feb 47(2):372-383. doi:10.1002/mrm.10051

23. Cerqueira MD, Weissman NJ, Dilsizian V et al (2002) Standardized myocardial segmentation and nomenclature for tomographic imaging of the heart. A statement for healthcare professionals from the Cardiac Imaging Committee of the Council on Clinical Cardiology of the American Heart Association. Circulation Jan 105(4):539-542. doi:10.1161/hc0402.102975

24. Lanza GA, Buffon A, Sestito A et al (Jan 2008) Relation between stress-induced myocardial perfusion defects on cardiovascular magnetic resonance and coronary microvascular dysfunction in patients with cardiac syndrome X. J Am Coll Cardiol 51(4):466-472. doi:10.1016/j.jacc.2007.08.060

25. Vinereanu D, Fraser AG, Robinson M, Lee A, Tweddel A (2002) Adenosine provokes diastolic dysfunction in microvascular angina. Postgrad Med J Jan 78(915):40-42. doi:10.1136/pmj.78.915.40

26. Mahfouz RA, Gouda M, Abdelhamid M (2020) Relation of microvascular dysfunction and exercise tolerance in patients with heart failure with preserved ejection fraction. Echocardiography Aug 37(8):1192-1198. doi:10.1111/echo.14799 
27. Lipkin DP, Canepa-Anson R, Stephens MR, Poole-Wilson PA (1986) Factors determining symptoms in heart failure: comparison of fast and slow exercise tests. Br Heart J May 55(5):439-445. doi:10.1136/hrt.55.5.439

28. Paulus WJ, Tschöpe C (Jul 2013) A novel paradigm for heart failure with preserved ejection fraction: comorbidities drive myocardial dysfunction and remodeling through coronary microvascular endothelial inflammation. J Am Coll Cardiol 62(4):263-271. doi:10.1016/j.jacc.2013.02.092

\section{Tables}

Table 1: Clinical and CMR characteristics in patients with non-ischemic cardiomyopathy vs the control cohort. 


\section{Clinical Characteristics}

\begin{tabular}{|c|c|c|c|c|}
\hline Age (years & & $51 \pm 13$ & $51 \pm 14$ & 0.95 \\
\hline Gender, N & & 18 (31\%) & $24(59 \%)$ & 0.006 \\
\hline Ethnicity & White, N (\%) & 52 (90\%) & $28(68 \%)$ & 0.02 \\
\hline & Black, N (\%) & $4(7 \%)$ & 12 (29\%) & \\
\hline & Hispanic, N (\%) & $1(2 \%)$ & $0(0 \%)$ & \\
\hline & Other, N (\%) & $1(2 \%)$ & $1(3 \%)$ & \\
\hline Diabetes, & & $7(12 \%)$ & $6(15 \%)$ & 0.77 \\
\hline Hypertens & & 25 (43\%) & 24 (59\%) & 0.16 \\
\hline Hyperlipid & V (\%) & $21(36 \%)$ & 16 (39\%) & 0.83 \\
\hline Atrial Fibr & /Flutter, N (\%) & $9(16 \%)$ & $3(7 \%)$ & 0.35 \\
\hline Creatinine & & $0.90 \pm 0.35$ & $0.93 \pm 0.23$ & 0.32 \\
\hline Hematocr & & $39 \pm 6$ & $38 \pm 6$ & 0.68 \\
\hline B-type $\mathrm{Na}$ & Peptide (ng/L) & $63(29-202)$ & 78 (39-991) & 0.39 \\
\hline Troponin- & & $0.01(0.01-0.02)$ & $0.01(0.0-0.05)$ & 0.17 \\
\hline CMR Cha & stics & & & \\
\hline LVEDD (cI & & $4.7(4.4-5.1)$ & $5.6(5.3-6.3)$ & $<0.001$ \\
\hline LVEDV (m & & $122(106-142)$ & $184(154-217)$ & $<0.001$ \\
\hline LVEDV Inc & $\left.\mathrm{mL} / \mathrm{m}^{2}\right)$ & $66 \pm 13$ & $94 \pm 29$ & $<0.001$ \\
\hline LVESV (m & & 45 (37-53) & $107(83-144)$ & $<0.001$ \\
\hline LVESV Inc & $\mathrm{mL} / \mathrm{m}^{2}$ ) & $22.8(20.0-28.0)$ & $55.2(41.4-68.6)$ & $<0.001$ \\
\hline LVEF (\%) & & $64 \pm 6$ & $42(32-45)$ & $<0.001$ \\
\hline LA Volum & red $\left(\mathrm{gm} / \mathrm{m}^{2}\right)$ & 39 (30-48) & $45(40-59)$ & 0.001 \\
\hline LV mass & & $83(64-102)$ & $110(86-130)$ & $<0.001$ \\
\hline LV mass I & $\left(\mathrm{gm} / \mathrm{m}^{2}\right)$ & $40.8(35.3-48.9)$ & $55.2(46.3-63.7)$ & $<0.001$ \\
\hline LGE prese & & $9(16)$ & $21(51)$ & $<0.001$ \\
\hline Number o & ients & $2(1-3)$ & $3(2-4)$ & 0.16 \\
\hline Myocardi & usion Slope (rest) & $\begin{array}{l}4.9(4.1-7.0) \\
\text { Page } 12 / 19\end{array}$ & $3.9(3.2-5.3)$ & 0.045 \\
\hline
\end{tabular}


Myocardial Perfusion Slope (stress)

Myocardial Perfusion Reserve Index

*LV: left ventricle; EDD: end diastolic dimension; EDV: end diastolic volume; EF: ejection fraction; ESV: end systolic volume; LA: left atrium; LGE: late gadolinium enhancement.

Table 2: Clinical and CMR characteristics in patients with vs. without impaired myocardial perfusion reserve index. 


\begin{tabular}{|c|c|c|c|}
\hline & $\begin{array}{l}\text { MPRI }<1.51 \\
(\mathrm{~N}=37)\end{array}$ & $\begin{array}{l}\text { MPRI } \geq 1.51 \\
(N=62)\end{array}$ & P-value \\
\hline \multicolumn{4}{|l|}{ Clinical Characteristics } \\
\hline Age (years) & $57(49-63)$ & $51(38-58)$ & 0.07 \\
\hline Gender, N (\% male) & $16(43 \%)$ & $26(42 \%)$ & 0.90 \\
\hline \multirow[t]{3}{*}{ Ethnicity } & $29(78)$ & $51(82)$ & 0.40 \\
\hline & $8(22)$ & $8(13)$ & \\
\hline & $0(0)$ & $1(2)$ & \\
\hline Other, N (\%) & $0(0)$ & $2(3)$ & \\
\hline Diabetes, N (\%) & $8(22)$ & $5(8)$ & 0.07 \\
\hline Hypertension, N (\%) & $25(68)$ & $24(39)$ & 0.005 \\
\hline Hyperlipidemia, N (\%) & $17(46)$ & $20(32)$ & 0.17 \\
\hline Atrial Fibrillation/Flutter, N (\%) & $4(11)$ & $8(13)$ & 0.76 \\
\hline Creatinine (mg/dL) & $0.84(0.73-1.05)$ & $0.82(0.74-1.02)$ & 0.66 \\
\hline Hematocrit (\%) & $37 \pm 5$ & $40 \pm 7$ & 0.06 \\
\hline B-type Natriuretic Peptide (ng/L) & $69(42-238)$ & $76(24-771)$ & 0.83 \\
\hline Troponin-I (ng/mL) & $0.01(0.00-0.05)$ & $0.01(0.008-0.01)$ & 0.82 \\
\hline \multicolumn{4}{|l|}{ CMR Characteristics } \\
\hline LVEDD (cm) & $5.3(4.6-5.8)$ & $4.9(4.5-5.4)$ & 0.09 \\
\hline LVEDV (mL) & $158(122-194)$ & $135(117-182)$ & 0.18 \\
\hline LVEDV Indexed $\left(\mathrm{mL} / \mathrm{m}^{2}\right)$ & $78(64-101)$ & $71(59-93)$ & 0.17 \\
\hline LVESV (mL) & $79(44-119)$ & $53(42-90)$ & 0.06 \\
\hline LVESV Indexed $\left(\mathrm{mL} / \mathrm{m}^{2}\right)$ & $39(23-60)$ & $28(21-39)$ & 0.06 \\
\hline LVEF (\%) & $47(35-62)$ & $59(49-65)$ & 0.02 \\
\hline LA Volume Indexed $\left(\mathrm{gm} / \mathrm{m}^{2}\right)$ & $45(35-51)$ & $39(31-48)$ & 0.14 \\
\hline LV mass (gm) & $106(80-118)$ & $88(64-109)$ & 0.04 \\
\hline LV mass Indexed $\left(\mathrm{gm} / \mathrm{m}^{2}\right)$ & $50(42-62)$ & $45(36-51)$ & 0.03 \\
\hline LGE presence (\%) & $14(37.8)$ & $16(26.2)$ & 0.23 \\
\hline Number of segments & $\begin{array}{l}2(2-3) \\
\text { Page } 14 / 19\end{array}$ & $3(2-4)$ & 0.47 \\
\hline
\end{tabular}


Myocardial Perfusion Slope (rest)

Myocardial Perfusion Slope (stress)
$5.2(3.4-7.8)$

$8.8(5.5-13.6)$
$4.4(3.4-6.1)$

0.17

0.048

*MPRI: myocardial perfusion reserve index; LV: left ventricle; EDD: end diastolic dimension; EDV: end diastolic volume; EF: ejection fraction; ESV: end systolic volume; LA: left atrium; LGE: late gadolinium enhancement.

Table 3: Multivariable linear regression for predicting myocardial perfusion reserve

\begin{tabular}{|c|c|c|c|c|}
\hline & $\begin{array}{l}\text { B coefficient } \\
(95 \% \mathrm{Cl})\end{array}$ & SE (B) & $P$ value & 95\% Cl (bootstrap)* \\
\hline $\mathrm{NICM}$ & $\begin{array}{l}-0.17 \\
(-0.30 \text { to }-0.04)\end{array}$ & 0.07 & 0.009 & -0.27 to -0.03 \\
\hline Age & $\begin{array}{l}-0.005 \\
(-0.009 \text { to }-0.0002)\end{array}$ & 0.002 & 0.04 & -0.008 to -0.0006 \\
\hline Male Gender & $\begin{array}{l}0.09 \\
(-0.04 \text { to } 0.22)\end{array}$ & 0.06 & 0.16 & -0.04 to 0.23 \\
\hline Diabetes & $\begin{array}{l}-0.10 \\
(-0.28 \text { to } 0.07)\end{array}$ & 0.09 & 0.25 & -0.26 to 0.15 \\
\hline Hypertension & $\begin{array}{l}-0.15 \\
(-0.28 \text { to }-0.03)\end{array}$ & 0.06 & 0.01 & -0.28 to -0.04 \\
\hline LGE & $\begin{array}{l}0.09 \\
(-0.053 \text { to } 0.239)\end{array}$ & 0.07 & 0.21 & -0.11 to 0.22 \\
\hline \multicolumn{5}{|c|}{$\begin{array}{l}\text { NICM: non-ischemic cardiomyopathy; LGE: late gadolinium enhancement } \\
\text { Log-transformation for myocardial perfusion reserve was performed } \\
\text { * } 2000 \text { bootstrap sample }\end{array}$} \\
\hline
\end{tabular}

\section{Figures}




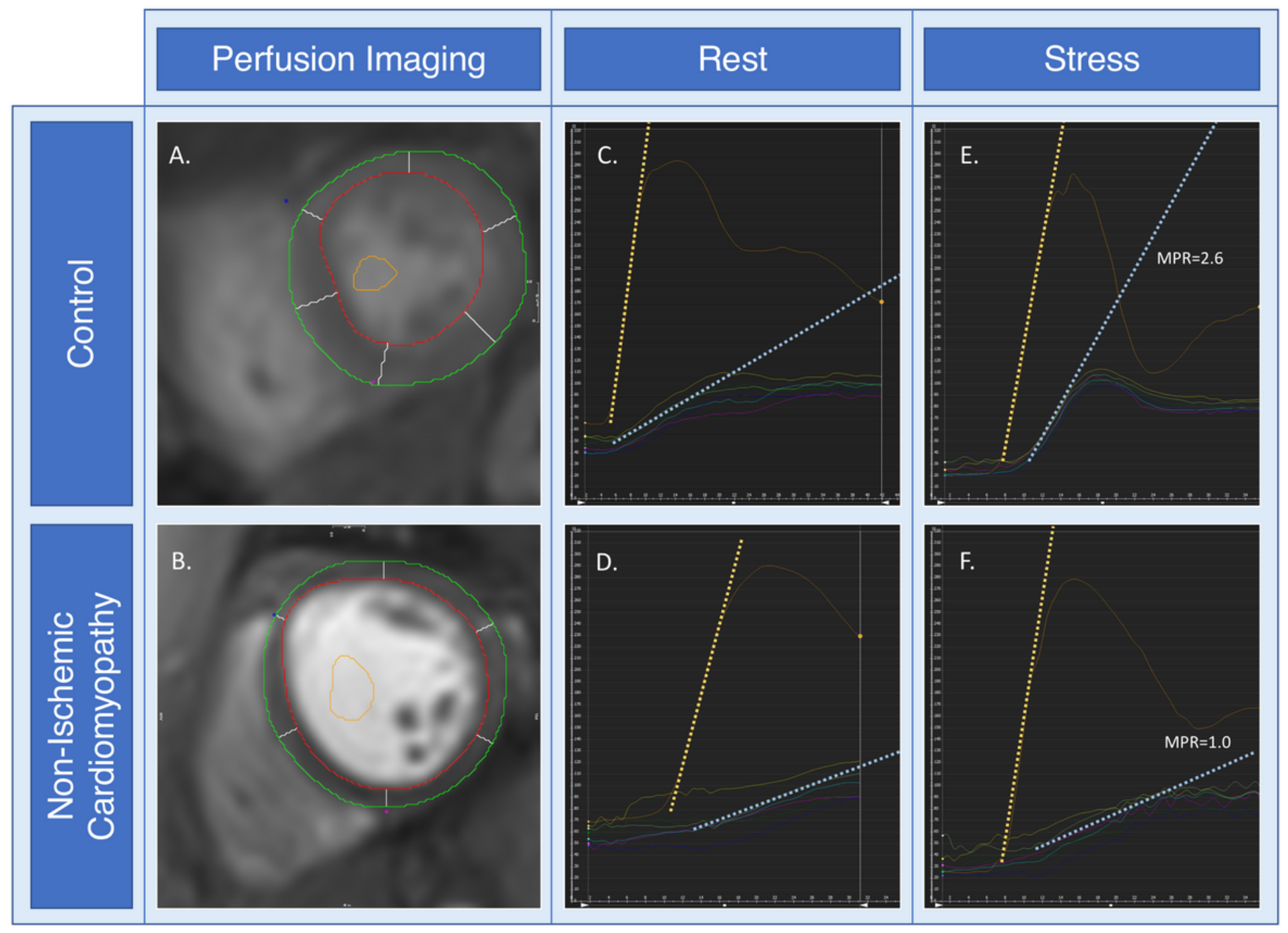

Figure 1

Determining Myocardial Perfusion Reserve Index. Panel A-B: First pass perfusion images with endocardial, epicardial, and blood pool contours. Panel C-F: Time intensity curve graphs at rest (C-D) and stress (E-F) first pass perfusion with maximal upslopes of blood pool (orange line) and myocardium (blue line). MPRI is calculated as the ratio of RUstress/RUrest where RU is ratio of maximal upslope of myocardium divided by blood pool. 


\section{Etiology of Non-Ischemic Cardiomyopathy $(n=41)$}

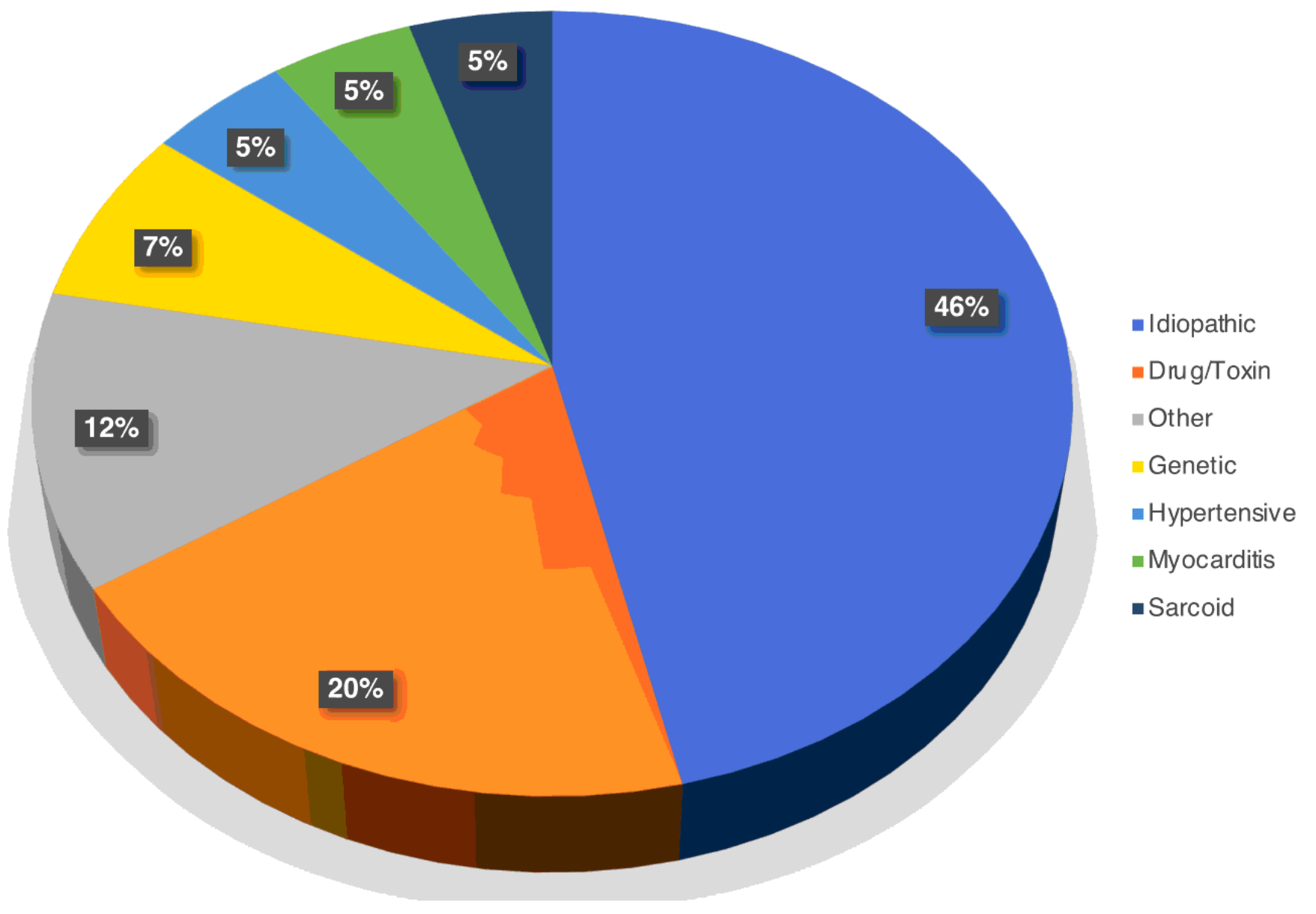

Figure 2

Etiologies of Non-Ischemic Cardiomyopathy 


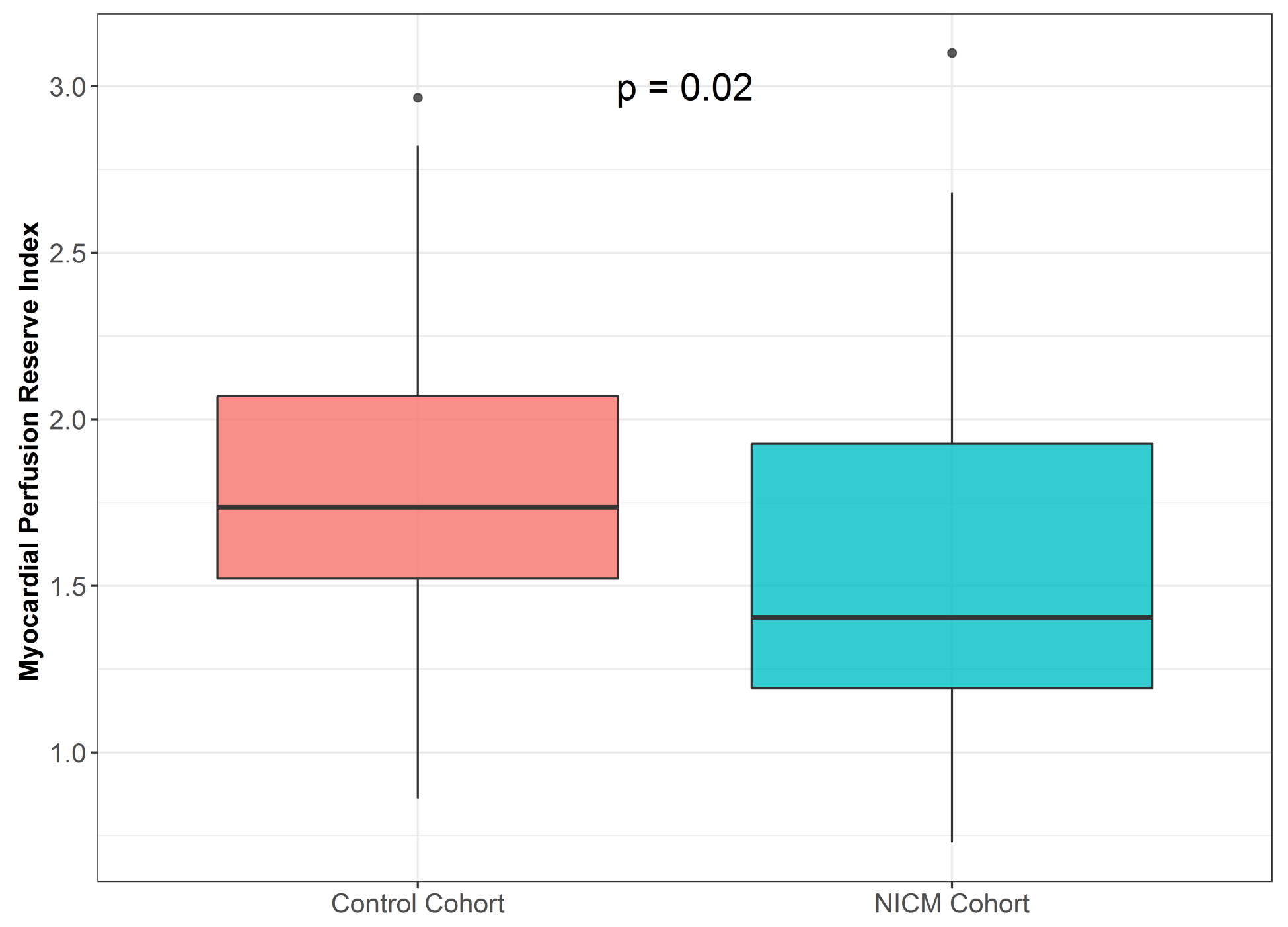

Figure 3

Myocardial perfusion reserve index (MPRI) in the non-ischemic cardiomyopathy (NICM) vs the control cohorts. Patients with NICM have significantly more impaired MPRI. 


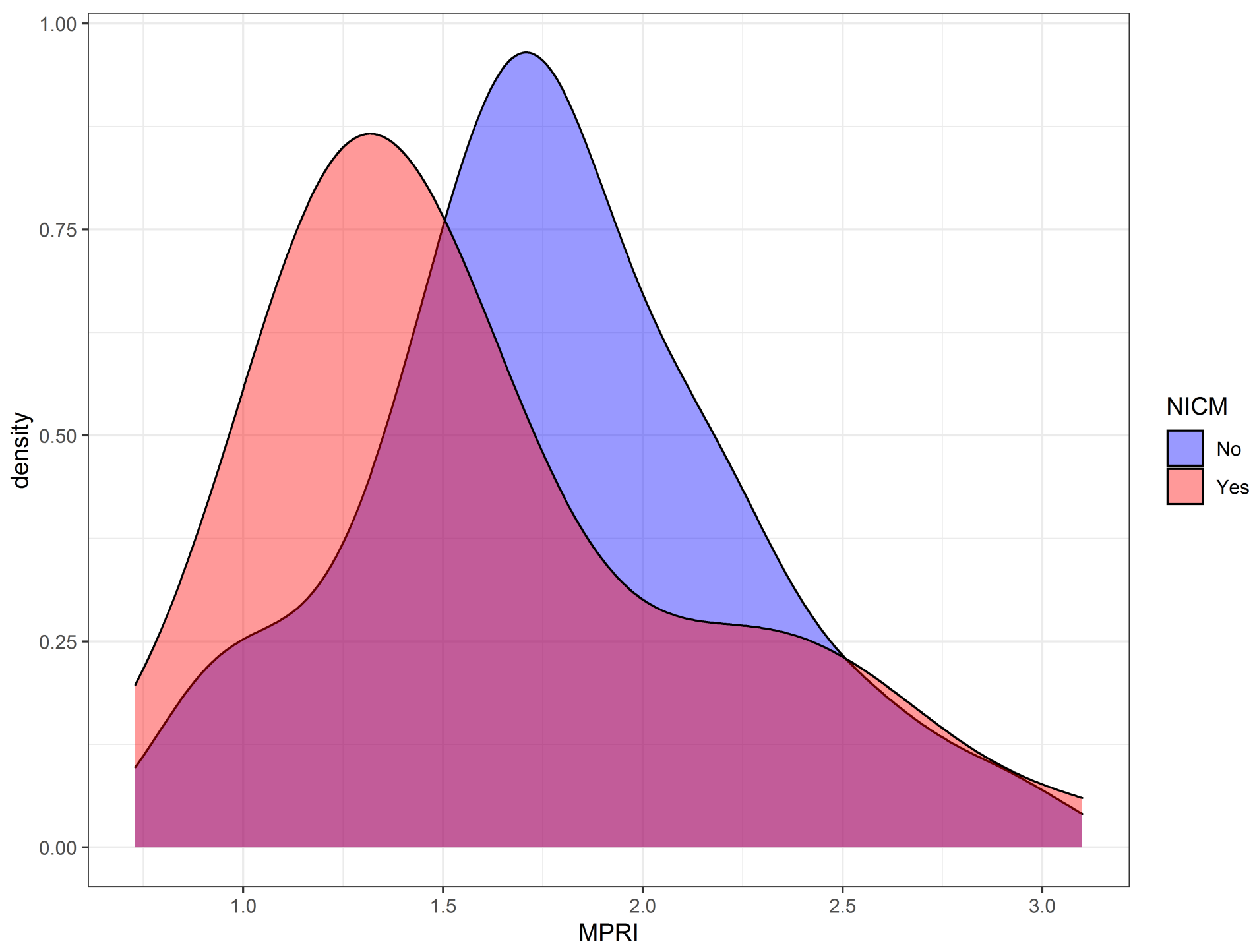

Figure 4

Density plot histogram displaying distributions of myocardial perfusion reserve index (MPRI) for nonischemic cardiomyopathy (pink) and control (blue) patients.

\section{Supplementary Files}

This is a list of supplementary files associated with this preprint. Click to download.

- MicrovascularSupplementalTableFinal.docx 